\title{
Microwave Assisted One-Pot Green Synthesis of Silver Nanoparticles Using Leaf Extracts from Vigna Unguiculate: Evaluation of Antioxidant and Antimicrobial Activities
}

\author{
Perera B.R., Kandiah M.*
}

BMS, School of Science, Sri Lanka

\begin{abstract}
Oxidative stress is caused by the increased levels of free radicals in the body which leads to various diseases. Antioxidants are known to reduce the risk of diseases caused by oxidative stress as they have the ability to donate electrons to these harmful free radicals. In addition to the natural antioxidants present in fruits and vegetables, synthetic antioxidants are also available. With the emergence of nanotechnology, the applications possessed by nanoparticles are limitless. One such application is in nanomedicine where nanoparticles are incorporated to study biological systems. The properties of the nanoparticles depend on the type of metal used and the method of synthesis. The use of plants for the production of silver nanoparticles (AgNPs) is key point of interest as it is rapid, eco-friendly, non-pathogenic and economical. Furthermore, this "Green synthesis" requires a single step for the biosynthesis of the nanoparticles as the silver ions are reduced and stabilized from the biomolecules present in the plant extract. In this study, cowpea leaf extracts were used for the on-pot green synthesis of silver nanoparticles to determine its antioxidant properties using phytochemical assays; Total flavonoid content (TFC), total phenolic content (TPC), total antioxidant activity (TAC), and free radical scavenging assays; Ferric reducing antioxidant properties (FRAP), ABTS and DPPH. The antibacterial activities were assessed by well diffusion method using gram positive (Staphylococus aureus) and gram negative (Escherichia coli) bacteria.
\end{abstract}

KEYWORDS: Silver Nanoparticles, Cowpea Leaf, Green Synthesis, Antioxidant, Antimicrobi

Corresponding author: Kandiah M, Email: mkandiah@gmail.com 


\section{INTRODUCTION}

Nano biotechnology is a branch of nanotechnology that incorporates the use of nanoparticles (NPs) to study biological systems. NPs lies in the nanoscale (1-100 $\mathrm{nm}$ ) and contains a surrounding interfacial layer consisting of organic and inorganic molecules and ions, which affects its properties. NPs formed by noble metals like platinum, gold and silver have been applied in a variety of fields such as information storage, electronics and in numerous medical and biological applications. Silver NPs (AgNPs) are of interest as they have been used in many biological applications which encounter the human system such as pharmaceutical and medical products due to its unique properties (Mohammadi, Pourseyedi, and Amini; Banerjee et al.) (Zhang et al.).

There are several methods to synthesize NPs. Techniques such as laser ablation, chemical reduction, photochemical reduction, thermal decomposition, electron irradiation, and gamma irradiation have various limitations such as the high cost, low stability of the NPs, production of toxic compounds that are harmful to the environment and the complexity of the procedure (Iravani et al.). Due to this reason, the green synthesis of NPs, which was first reported by Gardea-Torresdey and his colleagues, was developed (Gardea-Torresdey et al.). The green synthesis method uses reliable methods to develop safe and environment friendly NPs which were more stable (Banerjee et al.; Mohammadi, Pourseyedi, and Amini). Protocols for the biological synthesis of NPs included using plants, bacteria and fungi (Mohammadi, Pourseyedi, and Amini). However, using plant extracts for the synthesis of NPs is more advantageous over microorganisms as there is a reduced biohazard and is easy to make improvements. Furthermore, maintaining cell cultures are not necessary. Overall, the use of plant extracts for the synthesis of NPs have proven to be the safest and cost-effective method (Mohammadi, Pourseyedi, and Amini). In this project, silver was used to make NPs from extracts of the cowpea leaf as there are no toxic chemical involved and the extract provides natural capping agents for the stabilization of the AgNPs (Ahmed et al.).

Vigna unguiculata (L.) Walp, commonly referred to as cowpea, is a dicotyledon plant belonging to the Fabaceae family. It is a native crop from Africa commercially cultivated as an annual crop in both subtropical and tropical parts of the world (Igwe et al.; Carvalho et al.). The production level in countries like Brazil, Cuba, Ghana, Mozambique, Nigeria, Sri Lanka, Sudan, Zambia and Zimbabwe is increasing due to availability of improved cowpea varieties (Ngalamu, Odra, and Tongun).

Cowpea has the capacity to grow in low fertility soils and has adapted to high temperatures and drought and a wide range of soil $\mathrm{pH}$ which makes this grain legume the crop of interest in the African region as the crops face predicted environmental changes (e.g. increased temperature, reduction of water availability) associated with climate change (Carvalho et al.). Studies have shown that environmental factors play a role in the antioxidant activity of leaves of plants (Liu et al.).

Cowpea has been used in the human diet, as well as in forage for animal feeding. Cowpea seeds provide a rich source of food content with a large proportion of carbohydrates (53\%), 22-33\% (215 to $437 \mathrm{~g} \mathrm{~kg}-1)$ of protein and $2 \%$ of fat (Igwe et al.; Gonçalves et al.). The young leaves, fresh pods and fresh seeds are 
also consumed in some world regions where the protein content of the leaves have been reported to be within 203$394 \mathrm{~g} \mathrm{~kg}-1$ (Gonçalves et al.).

The antioxidant (Saraiva et al.) and antibacterial (Kritzinger et al.) properties of cowpea has been investigated in previous studies which makes it an ideal candidate to synthesize nanoparticles.

Normal cellular metabolism produces free radicals which are reactive chemicals with an unpaired electron in its outer valence. Environmental factors, such as air pollutants or cigarette smoke can also cause the formation of free radicals. Reactive oxygen species (ROS) are oxygen free radicals such as the hydroxyl radical $(\bullet \mathrm{OH})$, superoxide $(\mathrm{O} 2 \cdot-)$, singlet oxygen (1/2O2), and hydrogen peroxide (H2O2). These free radicals in low concentrations are beneficial to the human body assisting in cell maturation and assists in immune reactions against pathogens. In large concentrations, free radicals cause oxidative stress which leads to various diseases. (Hajhashemi et al.; Pham-Huy, He, and Pham-Huy; Birben et al.; Poljsak, Šuput, and Milisav).

Antioxidants are known to reduce the risk of diseases related to oxidative stress caused by ROS. Phenolic compounds and condensed tannin contents are commonly found in plants and have a potential role in disease prevention and health promotion, having multiple biological effects, including antibacterial, antiinflammatory, and antioxidant activity. Studies have shown a correlation of flavonoids and phenols in plants to antioxidant activities (Fidrianny, Puspitasari, and Marlia Singgih; Zia-UlHaq et al.).

Bacterial and fungal pathogens cause diseases in humans and animals. Plant extracts are used as an alternative way to control these pathogens as the current antimicrobial agents cause toxicity and bacteria can form drug resistant strains. Many of these plant extracts contain secondary compounds that have an inhibitory effect on harmful bacterial and fungal human pathogens (Ashraduzzaman et al.). Studies have shown the antimicrobial activity of cowpea root exudates(Nóbrega et al.) and leaf extracts (Kritzinger et al.).

The aim of this study is to synthesize silver nanoparticles and to determine its antioxidant and antibacterial activity against the water extracts of the samples. Antioxidant properties were analyzed using the total flavonoid content (TFC), total phenolic content (TPC), total antioxidant content (TAC), ferric reducing antioxidant properties (FRAP), ABTS and DPPH free radical scavenging assay. The antibacterial activity was determined using the well diffusion test for Staphylococcous aureus and Escherichia coli.

\section{MATERIALS}

\section{Sample Collection}

Vigna unguiculate leaf samples were collected from the Field Crops Research and Development Institute (FCRDI) located in Angunakolapelessa, Sri Lanka (608'50.7"N 8054'21.9"E). There are five recommended varieties for cultivation which are Waruni (CP1), Dhawala (CP2), Bombay (CP3), MICP01 (CP4) and MI-35 (CP5) whose leaves were used in this study (Figure 1 and Table 1).

\section{Chemical and Reagents}

2,2'-azinobis(3-ethylbenzothiazoline-6sulphonic acid)

(C18H18N4O6S4) (CAS30931-67-0), 
Acetic acid, Aluminium Chloride (AlCl3) (CAS-7446-70-0), Ammonium molybdate ([NH4]6Mo7O24.4H2O) (CAS-12054-85- 2), Ammonium persulfate ((NH4)2S2O8) (CAS-7727-540), Ethanol, 2,2-diphenyl-1picrylhydrazyl (DPPH), Ferric chloride, Folin Ciocalteu phenol reagent, Methanol (CH3OH) (CAS-67-56-1), Hydrochloric acid, McFarland solution, Mueller-Hinton agar powder, Sodium acetate, Sodium carbonate (Na2CO3) (CAS-497-19-8), Saline, Silver nitrate solution, Sodium hydroxide, Sodium nitrate, Sodium sulphate (Na2SO4) (CAS- 7757-82-6), Sulfuric acid (H2SO4) (CAS-7664-93-9), 2,4,6,-tri(2- pycridyl)-s-triazine reagent and 2,4,6-Tris(2-pyridyl)-s-triazine (TPTZ).

\section{Apparatus}

Fume hood (BIOBASE FH1000), Analytical weighing scale, micropipettes, hot air oven (meditry DHA-9053A), spectrophotometer (JENWAY 6305), Microwave (SINGER SMW20C2), weighing scale, water bath (GEMMYCO YCW-010E), refrigerator and autoclave

\section{Consumables}

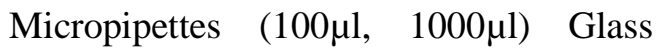
beakers $(50 \mathrm{~mL}, 100 \mathrm{~mL}$ and $250 \mathrm{~mL}$ ), measuring cylinders, filter funnels, falcon tubes ( $15 \mathrm{~mL}$ and $50 \mathrm{~mL}$ ), watch glasses, mortar and pestle, spatula, gauze, Whatman no.1 filter paper, sterile pipette tips, cuvettes, aluminium foil, petri plates (Plastic), test tubes, conical flasks (250 $\mathrm{mL}$ )

\section{METHODOLOGY}

All the experiments were conducted following good lab practice and using personal protective equipment. The respective $\mathrm{COSHH}$ and bioCOSHH forms were submitted prior to the commencement of the study.

Sample drying. The samples were dried in the shade for 4 days until there was no moisture was retained.

Sample extraction. $2 \mathrm{~g}$ of leaves in each type were measured using the balance, finely incised and placed in their individual beaker. $25 \mathrm{~mL}$ of distilled water was then added to each beaker, the mouth covered with foil and boiled for 60 minutes at $100{ }^{\circ} \mathrm{C}$. The extracts (mother liquid) were filtered through a muslin cloth and then by Whatman filter paper No. 1 using a funnel into $50 \mathrm{~mL}$ falcon tubes. These samples were kept at $4{ }^{\circ} \mathrm{C}$ until further use.

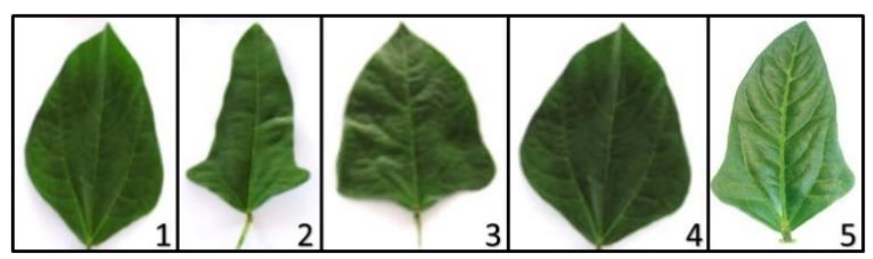

Figure 1. Cowpea leaf samples; 1) Waruni, 2) Dhawala, 3) Bombay 4) MICP-01 5) MI-35

Table 1: Varieties of leaves used in the study

\begin{tabular}{|l|l|l|}
\hline \multirow{2}{*}{ Sample } & \multicolumn{2}{|l|}{ Code name } \\
\cline { 2 - 3 } & Water Extracts & Nanoparticles \\
\hline Waruni & CP1 & CP1NP \\
\hline Dhawala & CP2 & CP2NP \\
\hline Bombay & CP3 & CP3NP \\
\hline MICP-01 & CP4 & CP4NP \\
\hline MI-35 & CP5 & CP5NP \\
\hline
\end{tabular}


Synthesis of silver nanoparticles.

$20 \mathrm{~mL}$ of $1 \mathrm{mM}$ silver nitrate solution was mixed with $1 \mathrm{~mL}$ of the mother liquid in each respective beaker. These solutions were then placed in a turntable microwave at MEDIUM power (511W) for 4 minutes discontinuously. The absorbance was measured from 300$500 \mathrm{~nm}$ using silver nitrate as a blank (Banerjee et al.).

Preparation of diluted samples.

For each extracted sample, a volume of 1 $\mathrm{mL}$ of the mother liquid was diluted with $14 \mathrm{~mL}$ of distilled water in a $15 \mathrm{~mL}$ falcon tube. Similarly, $1 \mathrm{~mL}$ of synthesized nanoparticles was diluted with $14 \mathrm{~mL}$ of distilled water in a $15 \mathrm{~mL}$ falcon tube. These samples were kept at 4 ${ }^{\circ} \mathrm{C}$ until further. The diluted samples were used for assays (unless stated) and were conducted in triplicates.

Determination of Total Flavonoid Content (TFC). The TFC was assessed by adding $1.5 \mathrm{~mL}$ of the diluted sample and $1.5 \mathrm{~mL}$ of $2 \% \mathrm{w} / \mathrm{v}$ aluminium chloride and incubated for 10 minutes at room temperature. The absorbance was measured at $415 \mathrm{~nm}$. The blank used for calibration was distilled water. The concentration was calculated for the equivalence of quercetin and expressed in mgQE/100g (Chang, Yang, and Wen; Nassourou et al.).

Determination of Total Phenolic Content (TPC).

The TPC was assessed by adding $0.5 \mathrm{~mL}$ of the diluted sample to a mixture of 2.5 $\mathrm{mL}$ of $10 \%$ Folin-Ciocalteu reagent and 2 $\mathrm{mL}$ of $7.5 \%$ sodium carbonate solution. The samples were then incubated at 40 ${ }^{\circ} \mathrm{C}$ for 30 minutes and the absorbance was measured at $765 \mathrm{~nm}$ using distilled water as blank. The concentration was calculated for the equivalence of gallic acid and expressed in mgGAE/100g (Saraiva et al.).

Determination of Total Antioxidant Content (TAC).

The TAC was assessed by adding $1 \mathrm{~mL}$ of prepared solution $(0.6 \mathrm{M}$ sulphuric acid, $28 \mathrm{mM}$ sodium sulphate and $4 \mathrm{mM}$ ammonium molybdate) to $3 \mathrm{~mL}$ of diluted sample and incubated at $90^{\circ} \mathrm{C}$ for 90 mins. Following incubation, the absorbance was measured at $695 \mathrm{~nm}$ using distilled water as blank. The concentration was calculated for the equivalence of ascorbic acid and expressed in mgAAE/100g (Kumar and Jain).

Ferric Reducing Antioxidant Properties (FRAP) assay.

The FRAP assay was carried out by adding $0.09 \mathrm{~mL}$ of diluted sample and $0.27 \mathrm{~mL}$ of distilled water to the respective cuvettes of each sample and placed in the spectrophotometer. $2.7 \mathrm{~mL}$ of FRAP solution was added to the cuvette placed in the spectrophotometer and the absorbance was measured at 595 $\mathrm{nm}$ per minute time for each sample (Kumar and Jain).

ABTS for water extract and silver nanoparticle samples. A mixture of 150 $\mu \mathrm{l}$ of diluted sample with $2850 \mu \mathrm{l}$ of ABTS solution (7 mM ABTS $+2.45 \mathrm{mM}$ ammonium persulfate; $3 \mathrm{~mL}$ of solution diluted with $100 \mathrm{~mL}$ of methanol) was added to each respective cuvette. The initial concentration of the ABTS working solution was noted and the absorbance of the samples were measured at $734 \mathrm{~nm}$ per minute interval using methanol as a blank (Shalaby and Shanab).

3.10 DPPH for water extracts and silver nanoparticle samples

$2 \mathrm{~mL}$ of DPPH solution was added to 1 $\mathrm{mL}$ of diluted sample and the absorbance 
was measured at $517 \mathrm{~nm}$ using ethanol as a blank (Nassourou et al.).

Optimization of the synthesis of silver nanoparticles.

All the five samples were prepared as mentioned in the synthesis of AgNPs and optimized at LOW (140 W), MEDIUM LOW (364 W) and MEDIUM (511 W) for 2.5 minutes, 4 minutes and 5.5 minutes, with one set left for overnight incubation in the dark at room temperature. The absorbances were measured for each optimization from 300-500 nm using $1 \mathrm{mM}$ AgNO3 as a blank.

Median Inhibition Concentration (IC50). A series of six concentrations, $100 \%$, $80 \%, 60 \%, 40 \%, 20 \%$ and $10 \%$ were prepared using $2850 \mu \mathrm{l}$ of ABTS solution, with varying volumes of distilled water and diluted sample according to the respective concentration. The absorbance of the prepared concentrated samples was then measured at $734 \mathrm{~nm}$ using methanol as the blank.

Well diffusion method for Staphylococcous aureus and Escherichia coli. $25 \mathrm{~mL}$ Muller-Hinton agar were poured to 20 sterilized petri dishes under a fume hood and left to solidify. Meanwhile, $1 \mathrm{~mL}$ of the original water extract and silver nanoparticle samples was added to individual watch glasses and air dried for 30 minutes. A saline solution of $0.9 \%$ was prepared and inoculated with Staphylococcous aureus and E. coli separately whilst using a 0.5 O.D. McFarland solution as standard to compare the turbidity.

Following solidification, the petri dishes were divided into four parts and labelled as $(+)$ ve,

(-)ve, S1 and S2 along with their sample code and respective bacterium. The inoculated saline solutions were then swabbed on the agar using cotton swabs. Three wells, for (+)ve, S1 and S2 were prepared using a sterilized pipette tip. These wells were then filled with the concentrated sample for S1 and S2, distilled water for (-)ve and a gentamycin disc was placed on the $(+)$ ve region. The petri dishes were then left in the incubator and the zones of inhibition measured.

Statistical Analysis. ONE-way ANOVA was generated by using Microsoft ${ }^{\circledR}$ Excel 2016 software and the correlation graphs were produced using IBM SPSS Statistics 23 software.

Scanning Electron Microscopy. Scanning Electron Microscopy (SEM) analysis was conducted at Sri Lankan Institute of Nanotechnology (SLINTEC), Homagama, using the Hitachi SU6600 SEM.

\section{RESULTS AND DISCUSSION}

SYNTHESIS OF SILVER NANOPARTICLES.

AgNPs are being used in several fields including the medical field due to their unique physical and chemical properties. The green synthesis of AgNPs have shown to have a high yield, solubility and stability. Furthermore they are rapid, ecofriendly, non-pathogenic, economical and non-toxic which is advantageous over the other methods(Zhang et al.).

In this project, a microwave mediated, one-pot green synthesis of AgNPs was demonstrated. The silver ions are reduced and stabilized from pure Ag+ ions to $\mathrm{Ag}(0)$ by the biomolecules present in the leaf extracts such as proteins, amino acids, enzymes, polysaccharides, alkaloids, tannins, phenolics, saponins, terpenoids and vitamins (Ahmed et al.). Furthermore, microwave assisted synthesis is promising compared to 
conventional methods due to the short reaction times and less energy consumption (Iravani et al.). The microwave mediated biosynthesis of AgNPs was successful as a change in colour was observed (Figure 2) which displays the formation of nanoparticles and spectrophotometric studies showed a well-defined peak in the visible region at $430 \mathrm{~nm}$ and $440 \mathrm{~nm}$ which corresponds to the surface plasmon resonance of AgNPs (Figure 3). These features are characteristic for the formation of AgNPs.

The optimization for the synthesis of AgNPs was carried out (Table 2) which showed that the optimal power of the microwave was MEDIUM LOW (364 W) for 4 minutes as it nanoparticles were formed in all samples. The characterization of the AgNPs was conducted by SEM analysis (Figure 4).

Prior to this method, the conventional method for the AgNP synthesis was carried, which was mixing the plant extract with AgNO3 and incubating in the oven at $100{ }^{\circ} \mathrm{C}$ and $60{ }^{\circ} \mathrm{C}$ for 30 minutes. This method produced an unstable colloid with aggregation and sedimentation and a change in colour, however, the spectrophotometric analysis showed no prominent peaks in the UV-vis spectrum. Characterization of the synthesized AgNPs is vital as the physiochemical properties of a particle may have a significant impact on their biological properties. The morphological studies, i.e., the shape and size of the particles, were clarified with the help of SEM. The analysis was carried out by placing aliquots of AgNPs samples on a carboncoated copper grid and allowed to dry. The sample underwent sputter coating which applied an ultra-thin coating of gold, which is an electrically-conducting metal. Sputter coating prevents charging of the specimen by increasing the conductivity (Anandalakshmi, Venugobal, and Ramasamy; Lin et al.). The sample was then placed in the SEM for analysis. The scans obtained displayed the spherical nature of the nanoparticles with a size of $40 \mathrm{~nm}$. The optical properties of the synthesized AgNPs were analyzed by UV-Visible absorbance spectroscopy. The conductivity of the synthesized AgNPs can be analyzed by measuring the band gap. The energy difference, measured in $\mathrm{eV}$, between the top of the valence band and bottom of the conduction band in insulators and semiconductors is referred to as the band gap. Band energy is the specific minimum amount of energy required for an electron to jump from a valence band to conduction band. Based on the band gap energies the materials can be classified as insulators $(>4 \mathrm{eV})$ and semiconductors $(<3 \mathrm{eV})$. The band gap energy can be calculated by the following equation, Band Gap Energy $€=$ $\mathrm{h} \times \mathrm{C} / \lambda$ where $\mathrm{h}$ is the Planck's constant $=$ $6.626910-34 \mathrm{~J} \mathrm{~s}, \mathrm{C}$ is the speed of light $=3 \times 108 \mathrm{~m} / \mathrm{s}$, and $\lambda$ is the cutoff wavelength which ranges from $420-460$ $\mathrm{nm}$ for the samples. The band gap energies and the classification of the samples are shown in table 3 which was calculated by the following Planck's equation (Sundeep et al.).

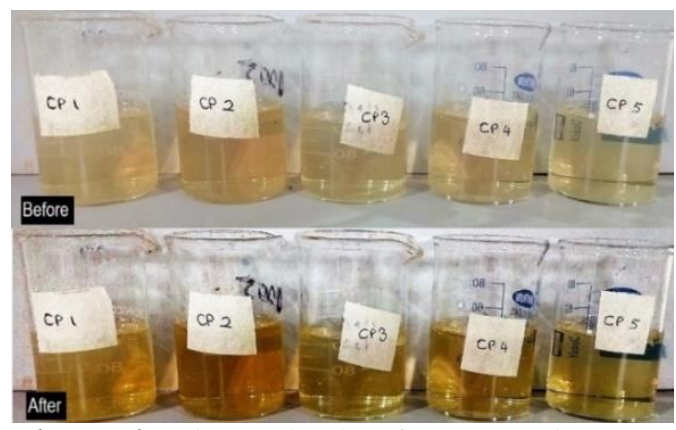

Figure 2. Biosynthesis of AgNP using cowpea leaf extracts. (Top) Silver Nitrate 
solution with cowpea leaf extract before heating. (Bottom) After heating

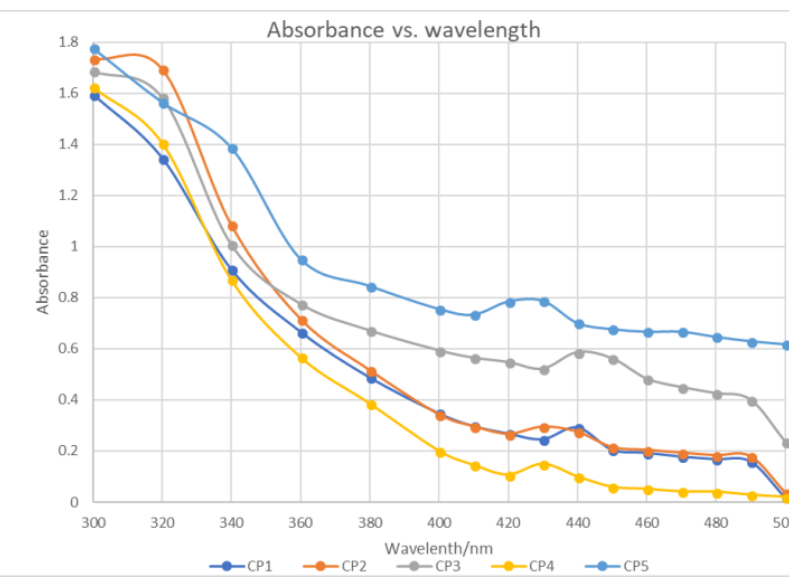

Figure 3. UV-vis spectrum of silver nanoparticles

Table 2: Optimisation of biosynthesis of AgNPs using different times and power

\begin{tabular}{|c|c|c|c|c|}
\hline \multirow[t]{2}{*}{ Sample } & \multicolumn{3}{|c|}{ Power } & \multirow{2}{*}{$\begin{array}{c}\text { Time } \\
\text { (minutes) }\end{array}$} \\
\hline & LOW & $\begin{array}{c}\text { MEDIUM } \\
\text { LOW }\end{array}$ & MEDIUM & \\
\hline \multirow[t]{3}{*}{ CP1 } & $x$ & $x$ & $x$ & 2.5 \\
\hline & $x$ & $\checkmark$ & $x$ & 4 \\
\hline & $x$ & $x$ & $x$ & 5.5 \\
\hline \multirow[t]{3}{*}{ CP2 } & $x$ & $x$ & $\checkmark$ & 2.5 \\
\hline & $x$ & $\checkmark$ & $x$ & 4 \\
\hline & $x$ & $x$ & $x$ & 5.5 \\
\hline \multirow[t]{3}{*}{ CP3 } & $x$ & $x$ & $\checkmark$ & 2.5 \\
\hline & $x$ & $\checkmark$ & $x$ & 4 \\
\hline & $x$ & $x$ & $x$ & 5.5 \\
\hline \multirow[t]{3}{*}{ CP4 } & $x$ & $x$ & $\checkmark$ & 2.5 \\
\hline & $x$ & $\checkmark$ & $\checkmark$ & 4 \\
\hline & $x$ & $x$ & $\checkmark$ & 5.5 \\
\hline \multirow[t]{3}{*}{ CP5 } & $x$ & $x$ & $\checkmark$ & 2.5 \\
\hline & $x$ & $\checkmark$ & $\checkmark$ & 4 \\
\hline & $x$ & $x$ & $\checkmark$ & 5.5 \\
\hline
\end{tabular}

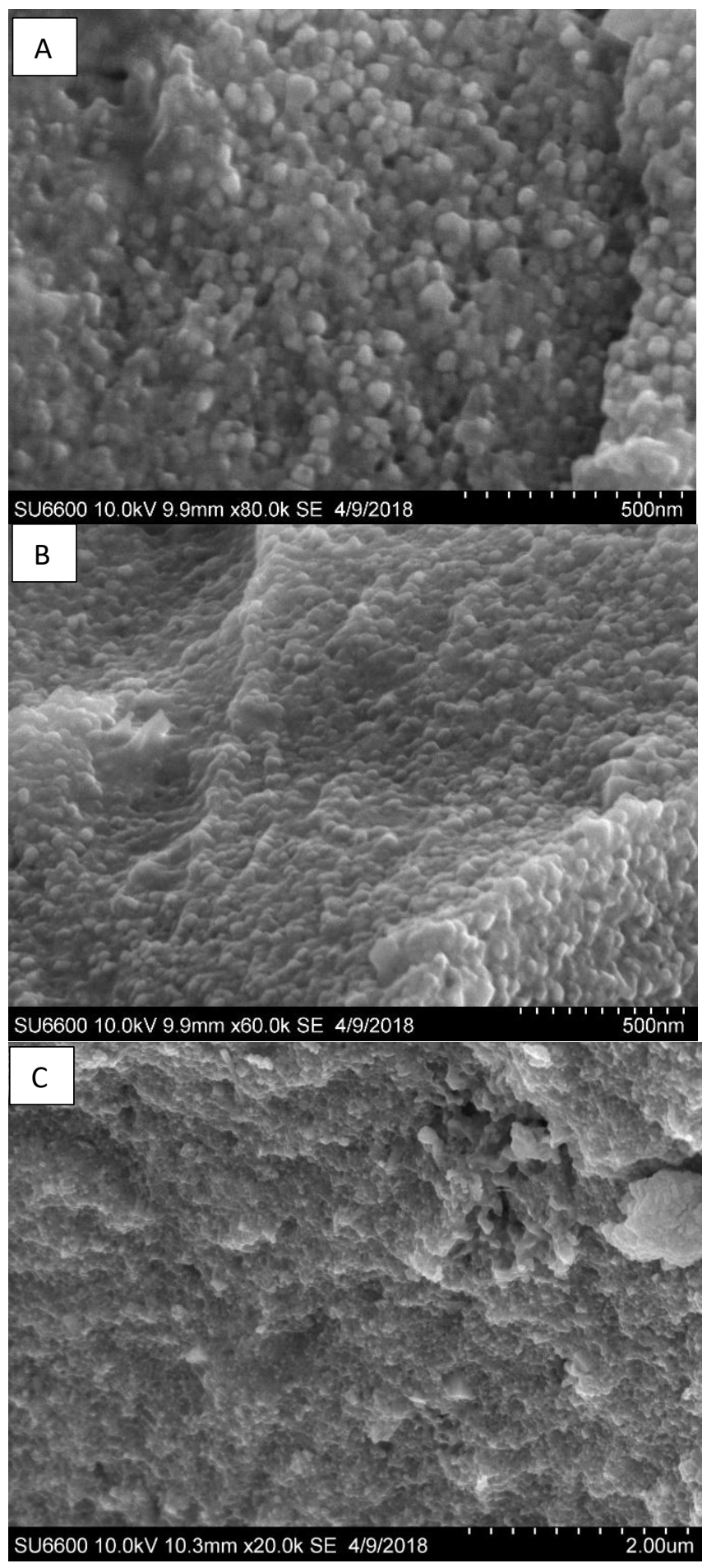

Figure 4. SEM images of the synthesized CP4NP AgNPs at A) 80.0k magnificence B) $60.0 \mathrm{k}$ magnificence C) $20.0 \mathrm{k}$ 
magnificence. AgNPs showing a spherical shape and a size of $40 \mathrm{~nm}$

Table 3. Classification of conduction in synthesised AgNPs

\begin{tabular}{|l|l|l|}
\hline Sample & $\begin{array}{l}\text { Band } \\
\text { Gap } \\
\text { Energy } \\
\mathbf{( e V )}\end{array}$ & Classification \\
\hline CP1NP & 2.9 & Semiconductor \\
\hline CP2NP & 2.9 & Semiconductor \\
\hline CP3NP & 2.8 & Semiconductor \\
\hline CP4NP & 2.9 & Semiconductor \\
\hline CP5NP & 2.8 & Semiconductor \\
\hline
\end{tabular}

Total Flavonoid Content.

The TFC (QE) was estimated by Aluminium chloride (AlCl3) colorimetric method. The AlCl3 forms acid stable complexes with the C-4 keto groups and either the C-3 or C-5 hydroxyl group of flavones and flavanols, having the maximum absorption at $415 \mathrm{~nm}$ (Bag and Devi; Chang, Yang, and Wen). The TFC of the water extracts was observed to be highest compared to AgNPs with CP2 being the highest followed by CP1 >CP4 $>$ CP3 >CP5 and CP4NP showing the highest value in the AgNPs followed by CP5NP >CP3NP >CP2NP>CP1NP, where CP2 shows a higher value between water extracts and AgNPs (Table 4). However, there's no statistical significance in the differences of their TFC ( $\mathrm{P}>0.05 / \mathrm{P}=0.072)$. This is further proved by the ONE-way ANOVA generated which shows a F-value $<$ Fcrit (F- 4.295564, Fcrit- 5.317655) indicating that there is no statistical significance between the groups. Compared to another study conducted by Saraiva and colleagues, more flavonoid content was seen compared to this study (Saraiva et al.).

The reason for the low TFC may be due to the AlCl3 binding to non-flavonoid molecules and/or the extraction of the sample which was carried out at $100{ }^{\circ} \mathrm{C}$ may have caused the degradation of the flavonoids (Maisuthisakul)

Total Phenolic Content.

The TPC (GAE) is determined by a colorimetric assay using the FolinCiocalteu reagent. The phenolic compounds react with the Folin-Ciocalteu reagent to form a blue colour complex that absorbs radiation which can be quantified at $765 \mathrm{~nm}$ (Pontis et al.). The TPC of the AgNPs were seen to be higher compared to the water extracts with CP2NP being the highest value in the AgNPs followed by CP3NP >CP5NP $>\mathrm{CP} 1 \mathrm{NP}>\mathrm{CP} 4 \mathrm{NP}$, and CP2 higher in water extracts followed by $\mathrm{CP} 1>\mathrm{CP} 4$ $>$ CP5 >CP3 with CP2NP being the highest in both groups (Table 4). There is a statistical significance between the TPC $(\mathrm{P}<0.05 / \mathrm{P}=0.04125)$ as the ONE-way ANOVA generated shows a Fvalue $>$ Fcrit $\quad($ F-5.317655, $\quad$ Fcrit0.475456). A study conducted by Zia-UlHaq and colleagues on cowpea seed extracts showed similar concentrations for the total phenols (Zia-Ul-Haq et al.).

\section{Total Antioxidant Content.}

The TAC (AAE) was evaluated by using the phosphomolybdenum method based on the reduction of Mo (VI) to Mo (V) by the antioxidant compounds and subsequent formation of a green phosphate/Mo (V) complex at acidic $\mathrm{pH}$ which can be measured at $695 \mathrm{~nm} 24$. Similar to the TPC, the TAC of the AgNPs were higher compared to the water extracts with CP2NP being the 
highest value in the AgNPs followed by CP3NP $>$ CP5NP $>$ CP1NP $>$ CP4NP, and $\mathrm{CP} 2$ higher in water extracts followed by CP1 >CP4 >CP5 >CP3 with CP2NP being the highest in both groups (Table 5).

The ONE-way ANOVA generated shows a F-value $>$ Fcrit (F-9.598282, Fcrit5.317655) indicating that there is a statistical significance between the groups $(\mathrm{P}<0.05 / \mathrm{P}=0.014708)$.

Table 4. Phytochemical assays for the samples

\begin{tabular}{|l|c|c|c|}
\hline Sample & \multicolumn{1}{l}{ TFC } & \multicolumn{1}{l}{ TPC } & \multicolumn{1}{l|}{ TAC } \\
(mgQE/100g) & $\begin{array}{l}\text { (mgGAE/100g) } \\
\text { (mgAAE/100g) }\end{array}$ \\
\hline CP1 & 896.4 & 392.7 & 25.1 \\
\hline CPNP1 & 145.8 & 282.9 & 48.2 \\
\hline CP2 & 1303.1 & 508.7 & 37.0 \\
\hline CPNP2 & 703.1 & 114.3 & 157.3 \\
\hline CP3 & 752.6 & 304.0 & 9.7 \\
\hline CPNP3 & 520.8 & 885.7 & 90.9 \\
\hline CP4 & 896.4 & 382.4 & 19.2 \\
\hline CPNP4 & 822.9 & 368.6 & 45.5 \\
\hline CP5 & 709.9 & 330.0 & 11.9 \\
\hline CPNP5 & 718.8 & 1085.7 & 83.6 \\
\hline
\end{tabular}

FRAP assay. The FRAP assay used to determine the antioxidant capacity is based on the reduction of Fe(III) to Fe(II) by antioxidants in the presence of TPTz ligand forming a blue coloured complex with Fe(II) which can be measured at 595 nm (Apak et al.). The highest reducing power for the water extracts (Figure 5) was seen by CP1 at 5 minutes followed by CP2 and CP5 (7 minutes), CP3 (10 minutes) and CP4 (11 minutes). Similarly, the highest reducing power of the AgNPs (Figure 6) was seen by CP3NP and CP5NP at 2 minutes followed by CP4NP (5 minutes), CP1NP (8 minutes) and CP2 (10 minutes). The highest reducing power activity of the water extracts was observed in CP4 at $1665.55 \mathrm{mg}$ AAE/100 g at 11 minutes and the lowest in CP1 at $1637.45 \mathrm{mg}$ AAE/100 $\mathrm{g}$ at 5 minutes. Likewise, the highest reducing power activity of the AgNPs was observed in CP2NP at $1431.55 \mathrm{mg}$ AAE/100 g at 10 minutes and the lowest in CP3NP at $1411.64 \mathrm{mg}$ $\mathrm{AAE} / 100 \mathrm{~g}$ at 5 minutes. A study conducted by Perera and his colleagues on the same variety of cowpea samples showed a similar trend in the FRAP concentration compared to this study (Table 5) (Perera et al.)

ABTS assay.

The ABTS assay is based on the ABTS radical cation decolorization from a blue green colour to colourless caused by the scavenging activity of the antioxidants which can be measured at $734 \mathrm{~nm}$ (Shalaby and Shanab). In the water extracts, the highest scavenging activity was seen in CP2 where it reached 100\% in 40 minutes and the lowest activity was seen in CP3 where it was at $90.2 \%$ at 60 minutes (Figure 7).

The scavenging activity of CP2 can be related to the high TFC, TPC and TAC of the sample. For the AgNPs, the highest activity was observed in CP3NP where an activity of $40.9 \%$ was reached in 60 minutes and the lowest was CP4NP where its activity was $8.1 \%$ at 60 minutes (Figure 8). A similar pattern was observed in a study conducted by Saraiva and colleagues on cowpea cultivar extracts (Saraiva et al.)

\section{DPPH assay.}

The DPPH assay is based on the ability of 2,2-diphenyl-1- picrylhydrazyl, which is a stable free radical, deep purple in colour that is decolourized by antioxidants. This can be measured at $517 \mathrm{~nm}$ (Bag and 
Devi). Antioxidants have the ability to donate an electron to the DPPH free radical causing a colour change from purple to yellow indicating the decrease in the DPPH radical absorbance (Jadid et al.).

The overall highest DPPH inhibition activity was observed in the water extracts with CP1 showing the highest initial activity (88.8\%). The lowest initial activity was observed in CP2 (75.1\%) as well. The highest initial antioxidant activity in AgNPs can be observed in CP3NP (60.6\%) and the lowest in CP1NP (34.6\%) (Figure 9). A study conducted by Perera and colleagues on cowpea seed extracts showed similar for the DPPH activity (Perera et al.).

The reason to why the nanoparticles showed a lower free radical scavenging activity is possibly due to the electrons on the AgNPs reacting with the free radicals.

\section{IC50}

The IC50 value was calculated to determine the concentration of the sample required to inhibit $50 \%$ of radical (Jadid et al.). Samples with high antioxidant activities have a lower the IC50. As observed in Figure 10 and Table 6, the lowest IC50 is seen in AgNPs with the highest antioxidant activity present in CP5NP followed by CP3NP >CP4NP $>$ CP2NP >CP1NP. The highest antioxidant activity in water extracts is observed in CP3 followed by CP2 >CP1 $>$ CP5 >CP4 (Figure 11 and Table 6).

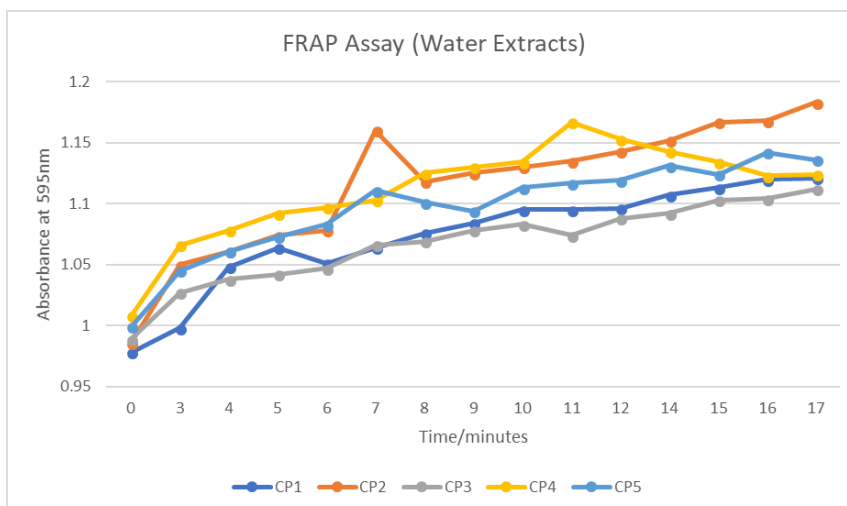

Figure 5. FRAP assay for water extracts

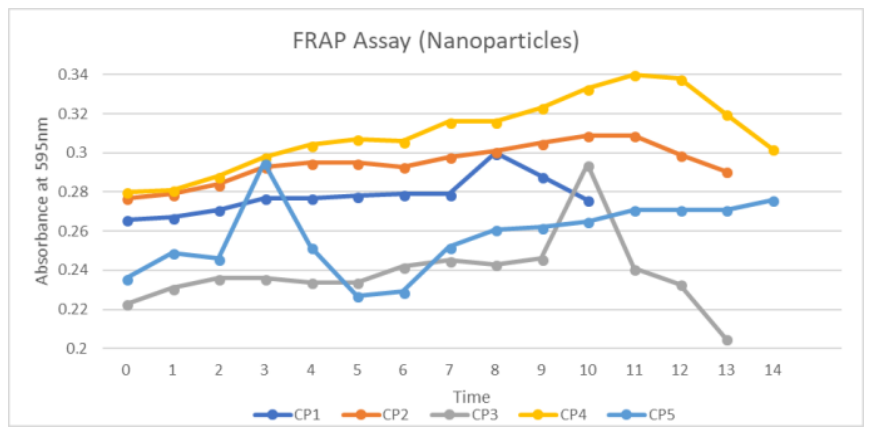

Figure 6. FRAP assay for nanoparticles

Table 5. Comparison of FRAP concentrations (Perera et al.)

\begin{tabular}{|c|c|c|}
\hline Sample & $\begin{array}{c}\text { FRAP }(\boldsymbol{\mu m o l} / \mathbf{g}) \\
\text { (Perera } \text { et } \boldsymbol{a l})\end{array}$ & $\begin{array}{c}\text { FRAP }(\mathbf{m g} \\
\text { AAE/100 } \mathbf{g})\end{array}$ \\
\hline $\mathbf{C P 1}$ & 35.28 & 1637.455 \\
\hline $\mathbf{C P} 2$ & 12.36 & 1663.636 \\
\hline $\mathbf{C P} 3$ & 24.07 & 1642.636 \\
\hline $\mathbf{C P} 4$ & - & 1665.545 \\
\hline $\mathbf{C P} 5$ & 10.94 & 1650.273 \\
\hline
\end{tabular}




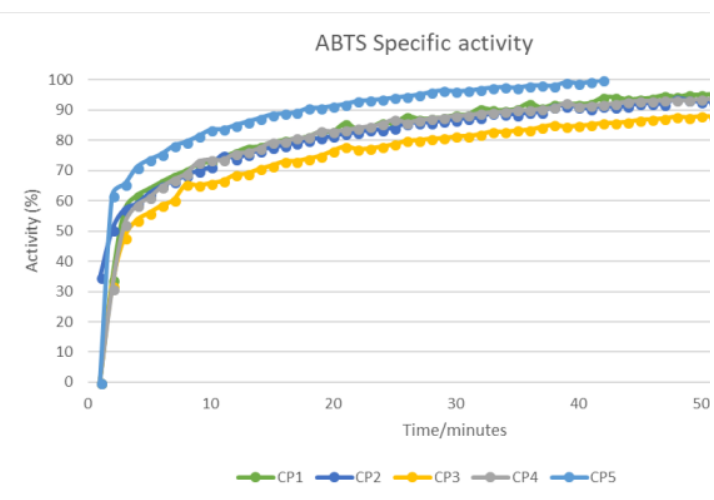

Figure 7. ABTS inhibition activity graph for water extracts

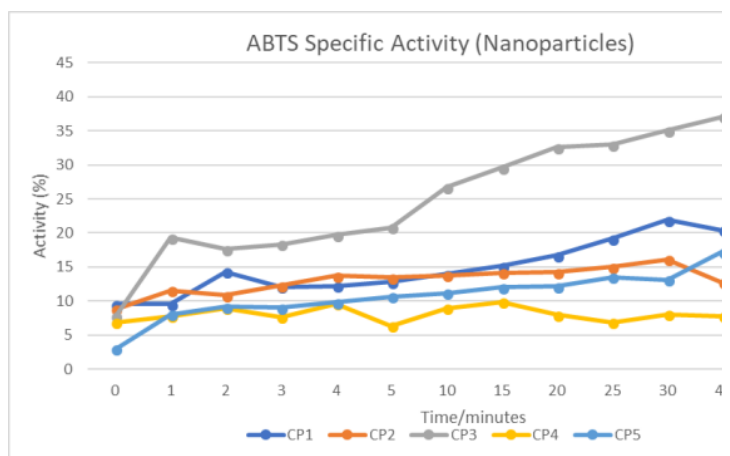

Figure 8. ABTS inhibition activity graph for nanoparticles

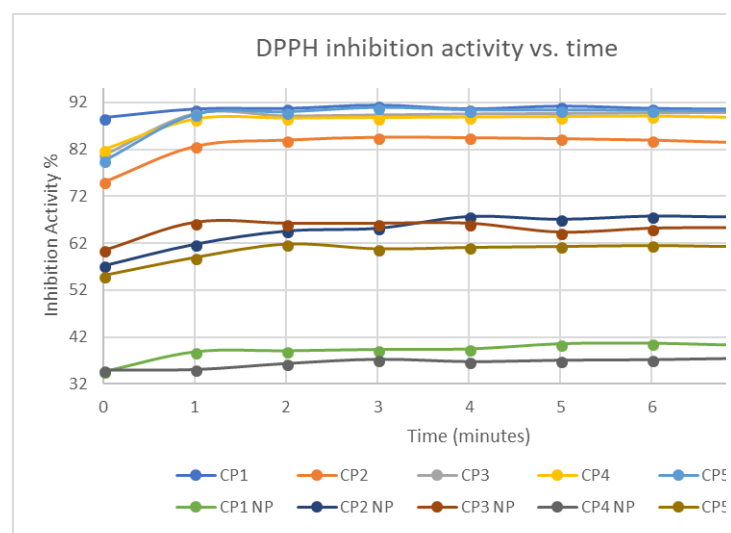

Figure 9. DPPH inhibition activity against time

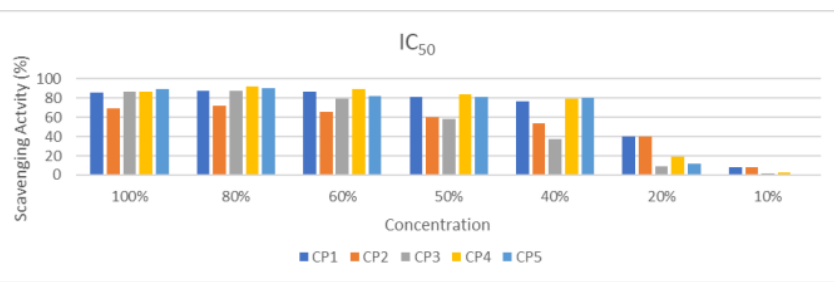

Figure 10. Inhibition concentration of the cowpea water extracts

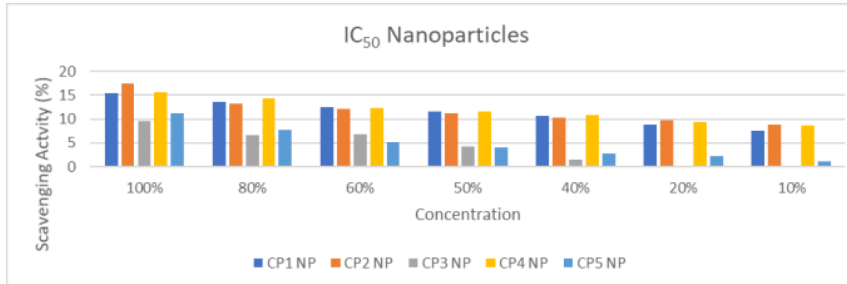

Figure 11. Inhibition concentration of the synthesized nanoparticles

Table 6. $\mathrm{IC}_{50}$ of the samples expressed in its specific activity (\%)

\begin{tabular}{|l|l|l|}
\hline & $\begin{array}{l}\text { Water } \\
\text { Extracts }\end{array}$ & AgNPs \\
\hline CP1 & 81.37369 & 11.65147 \\
\hline CP2 & 60.41909 & 11.2462 \\
\hline CP3 & 58.32363 & 4.207921 \\
\hline CP4 & 83.81839 & 11.65147 \\
\hline CP5 & 81.37369 & 3.960396 \\
\hline
\end{tabular}

Antimicrobial activity. The mechanism of action of AgNPs as antibacterial agents are still undiscovered however several hypotheses have been put forward. One hypothesis being that the cell walls of the Gram-negative bacteria were penetrated by the AgNPs which leads to increased cell permeability and death. Another theory is that the AgNPs cause membrane damage due to the formation of free radicals. Another concept is inhibition of bacterial growth and death by the binding of thiol groups and phosphorouscontaining bases, such as vital enzymes and DNA bases, to the Ag+. It has also been postulated that the size and shape of 
the AgNP affect the antibacterial activity. An increased surface-area-to-volume which allows for more interactions with the bacteria can be achieved with decreasing size. Furthermore, the shape of the AgNPs also play an important role in the antibacterial activity as there are shape dependant interactions with Gram negative bacteria (Park; Zhang et al.)

The antimicrobial study revealed that the zone of inhibition (ZOI) in $\mathrm{cm}$ was greater in the water extracts compared to the AgNPs for Staphylococcus aureus except for CP1 and CP5 (Figures 12, 13). A ONE-way ANOVA conducted for Staphylococcus aureus showed a Fvalue $<$ F-crit $\quad$ (F-0.783972, Fcrit5.317655) indicating that there is no significance difference between the ZOI of the samples. The ZOI for Escherichia coli however showed a greater ZOI for AgNPs compared to water extracts (Figures 14, 15). The ONE-way ANOVA for Escherichia coli showed a F-value $>\mathrm{F}$ crit (F-11.70149, Fcrit-5.317655) indicating that there is a significance difference between the ZOI of the samples.

The ONE-way ANOVA between the two bacteria shows an F-value $<$ F-crit (F2.551679, Fcrit-4.413873) indicating that there is no significant difference between the ZOI of S. aureus and E. coli.

\section{Statistical analysis}

The Pearson correlation factor (PCF) was used to determine the statistical relationship between tests. A strong relationship can be observed between ABTS and FRAP assays ( $\mathrm{R} 2$ value = 0.999) (Appendix 9.7). Similarly, a strong relationship is observed between the ABTS and DPPH and DPPH and FRAP. The correlation of the TAC assay with TPC and TFC assays were poor with a R2 value of 0.018 and 0.055 respectively.
Almost no relationship was seen in the TPC and TFC assay. The cause is assumed to be due to the $\mathrm{AlCl} 3$ binding to non flavonol compounds. The correlation between the assays are summarized in figure 16 .

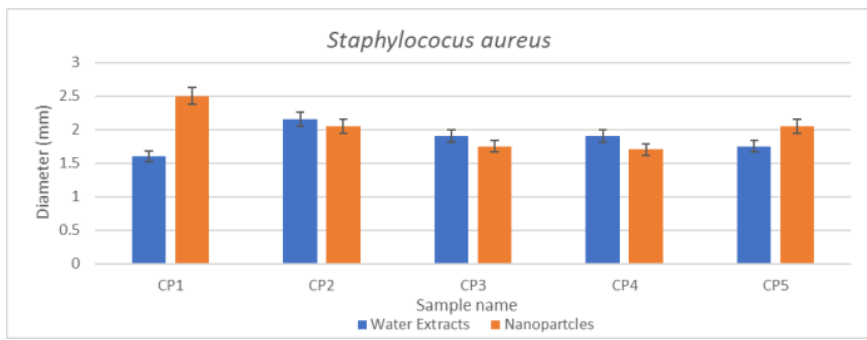

Figure 12. Inhibition zones of Staphylococcus aureus seen in MullerHinton agar plates



Figure 13. Zones of inhibition (ZOI) of S. aureus in CP1 (A) and CP5 (B) in water extracts (left) and AgNPs (right) 


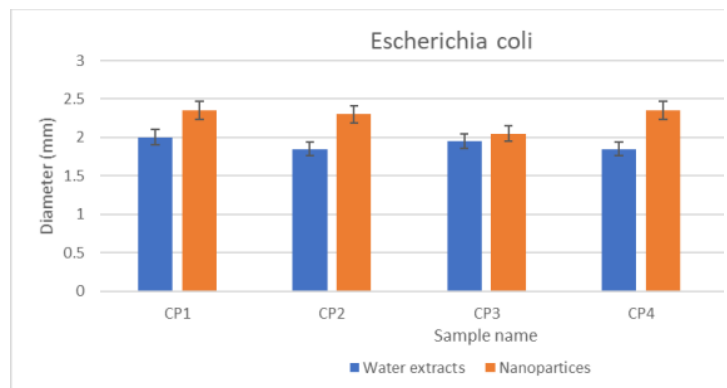

Figure 14. Inhibition zones of

Escherichia coli seen in Muller-Hinton agar plates

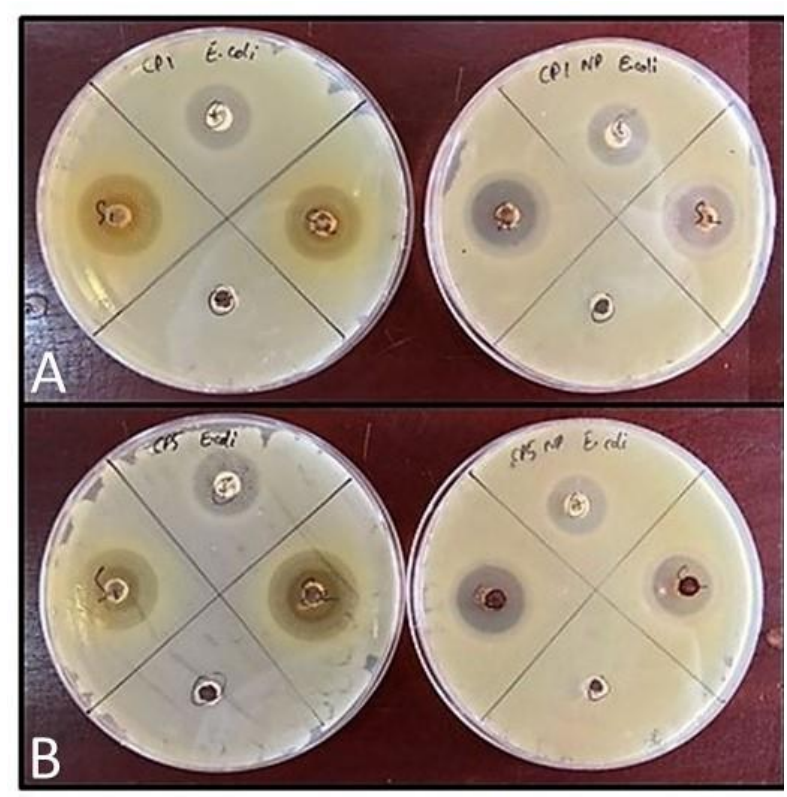

Figure 15. ZOI of E. coli in CP1 (A) and CP5 (B) in water extracts (left) and AgNPs (right)

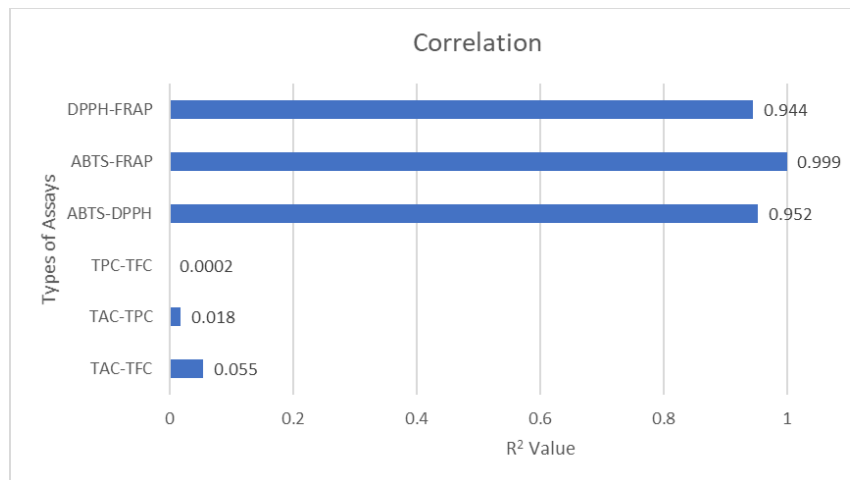

Figure 16. Correlation between assays

\section{CONCLUSIONS}

In conclusion, eco-friendly AgNPs can be synthesised using cowpea leaf extracts via a microwave assisted biosynthesis. The optimum power was discovered to be MEDIUM LOW (364 W) at 4 minutes. The nanoparticles showed higher antioxidant properties compared to the water extracts. Furthermore, the nanoparticles showed a greater ZOI for E. coli compared to S. aureus. Therefore, these AgNPs can be used as therapeutic agents in the alleviation of free radical mediated diseases.

\section{Conflicts of interest}

There are no conflicts to declare.

\section{Acknowledgements}

Authors thank Business Management School (BMS) for financial assistance.

\section{REFERENCES}

Ahmed, Shakeel et al. "A Review on Plants Extract Mediated Synthesis of Silver Nanoparticles for Antimicrobial Applications: A Green Expertise." Journal of Advanced Research 7.1 (2016): 17-28. Web. 
Anandalakshmi, K., J. Venugobal, and V. Ramasamy. "Characterization of Silver Nanoparticles by Green Synthesis Method Using Pedalium Murex Leaf Extract and Their Antibacterial Activity." Applied Nanoscience 6.3 (2016): 399408. Web.

Apak, Reşat et al. "Antioxidant Activity/Capacity Measurement. 1. Classification, Physicochemical Principles, Mechanisms, and Electron Transfer (ET)-Based Assays." Journal of Agricultural and Food Chemistry 64.5 (2016): 997-1027. Web.

Ashraduzzaman, Mohammad et al. "Antimicrobial Activity of Vigna Unguiculata L . Walp Seed Oil." 2 (2016): 70-75. Print.

Bag, G C, and P Grihanjali Devi. "Research Article Assessment of Total Flavonoid Content and Antioxidant Activity of Methanolic Rhizome Extract of Three Hedychium Species of Manipur Valley 1." International Journal of Pharmaceutical Sciences Review and Research 30.28 (2015): 154-159. Print.

Banerjee, Priya et al. "Leaf Extract Mediated Green Synthesis of Silver Nanoparticles from Widely Available Indian Plants: Synthesis, Characterization, Antimicrobial Property and Toxicity Analysis." Bioresources and Bioprocessing 1.1 (2014): 3. Web.

Birben, Esra et al. "Oxidative Stress and Antioxidant Defense." WAO Journal 5.January (2012): 9-19. Web.

Carvalho, Márcia et al. "Cowpea: A Legume Crop for a Challenging Environment." Journal of the Science of Food and Agriculture 97.13 (2017): 4273-4284. Web.
Chang, Chia-Chi, Ming-Hua Yang, and Hwei-Mei Wen. "Estimation of Total Flavonoid Content in Propolis by Two Complementary Colorimetric Methods." Journal of Food and Drug Analysis 10.3 (2002): 178-182. Web.

Fidrianny, Irda, Nuraini Puspitasari, and W. Marlia Singgih. "Antioxidant Activities, Total Flavonoid, Phenolic, Carotenoid of Various Shells Extracts from Four Species of Legumes." Asian Journal of Pharmaceutical and Clinical Research 7.4 (2014): 42-46. Print.

Gardea-Torresdey, J L et al. "Formation and Growth of $\mathrm{Au}$ Nanoparticles inside Live Alfalfa Plants." Nano Letters 2.4 (2002): 397-401. Web.

Gonçalves, Alexandre et al. "Cowpea (Vigna Unguiculata L. Walp), a Renewed Multipurpose Crop for a More Sustainable Agri-Food System: Nutritional Advantages and Constraints." Journal of the Science of Food and Agriculture 96.9 (2016): 2941-2951. Web.

Hajhashemi, V. et al. "Are Antioxidants Helpful for Disease Prevention?" Research in Pharmaceutical Sciences 2010: 5-12. Print.

Igwe, David Okeh et al. "Assessment of Genetic Diversity in Vigna Unguiculata L. (Walp) Accessions Using Inter-Simple Sequence Repeat (ISSR) and Start Codon Targeted (SCoT) Polymorphic Markers." BMC Genetics 18.1 (2017): 1-13. Web.

Iravani, S. et al. "Synthesis of Silver Nanoparticles: Chemical, Physical and Biological Methods." Research in Pharmaceutical Sciences 9.6 (2014): 385-406. Print. 
Jadid, Nurul et al. "Antioxidant Activities of Different Solvent Extracts of Piper Retrofractum Vahl . Using DPPH Assay." 020019 (2017): n. pag. Web.

Kritzinger, Q et al. "Antimicrobial Activity of Cowpea ( Vigna Unguiculata ) Leaf Extracts." South African Journal of Botany 71.1 (2005): 45-48. Web.

Kumar, Tekeshwar, and Vishal Jain. "Appraisal of Total Phenol, Flavonoid Contents, and Antioxidant Potential of Folkloric Lannea Coromandelica Using In Vitro and In Vivo Assays." Scientifica 2015 (2015): 1-13. Web.

Lin, Ping Chang et al. "Techniques for Physicochemical Characterization of Nanomaterials." Biotechnology Advances 32.4 (2014): 711-726. Web.

Liu, Wei et al. "Influence of Environmental Factors on the Active Substance Production and Antioxidant Activity in Potentilla Fruticosa L . and Its Quality Assessment." June (2016): 1-18. Web.

Maisuthisakul, Pitchaon. "Antioxidant Potential and Phenolic Constituents of Mango Seed Kernel from Various Extraction Methods." Kasetsart Journal Natural Science 43.5 SUPPL. (2009): 290-297. Print.

Mohammadi, Soheila, Shahram Pourseyedi, and Abbas Amini. "Green Synthesis of Silver Nanoparticles with a Long Lasting Stability Using Colloidal Solution of Cowpea Seeds (Vigna Sp. L)." Journal of Environmental Chemical Engineering 4.2 (2016): 2023-2032. Web.

Nassourou, Maina Antoine et al. "Genetics of Seed Flavonoid Content and
Antioxidant Activity in Cowpea (Vigna Unguiculata L. Walp.).” Crop Journal 4.5 (2016): 391-397. Web.

Ngalamu, Tony, James Odra, and Nixon Tongun. COWPEA PRODUCTION HANDBOOK. N.p., 2015. Print.

Nóbrega, F M et al. "Antimicrobial Proteins from Cowpea Root Exudates: Inhibitory Activity against Fusarium Oxysporum and Purification of a Chitinase-like Protein." Plant and Soil 272.1 (2005): 223-232. Web.

Park, Youmie. "A New Paradigm Shift for the Green Synthesis of Antibacterial Silver Nanoparticles Utilizing Plant Extracts." Toxicological Research 30.3 (2014): 169-178. Web.

Perera, O S et al. "Modulating Effects of Cowpea Incorporated Diets on Serum Lipids and Serum Antioxidant Activity in Wistar Rats." 44.1 (2016): 69-76. Print.

Pham-Huy, Lien Ai, Hua He, and Chuong Pham-Huy. "Free Radicals, Antioxidants in Disease and Health." International journal of biomedical science: IJBS 4.2 (2008): 89-96. Web.

Poljsak, Borut, Dušan Šuput, and Irina Milisav. "Achieving the Balance between ROS and Antioxidants: When to Use the Synthetic Antioxidants." Oxidative Medicine and Cellular Longevity 2013 (2013): n. pag. Web.

Pontis, Jonierison Alves et al. "Color, Phenolic and Flavonoid Content, and Antioxidant Activity of Honey from Roraima, Brazil." Food Science and Technology 34.1 (2014): 69-73. Web.

Saraiva, Regilda et al. "Identification and Quantification of Antioxidant 
Compounds in Cowpea 1 Identificação e Quantificação de Compostos Antioxidantes Em Feijão-Caupi." 5 (2017): 799-805. Web.

Shalaby, Emad A., and Sanaa M M Shanab. "Comparison of DPPH and ABTS Assays for Determining Antioxidant Potential of Water and Methanol Extracts of Spirulina Platensis." Indian Journal of Marine Sciences 42.5 (2013): 556-564. Print.

Sundeep, Dola et al. "Green Synthesis and Characterization of Ag Nanoparticles from Mangifera Indica Leaves for Dental Restoration and Antibacterial Applications." Progress in Biomaterials 6.1-2 (2017): 57-66. Web.

Zhang, Xi-Feng et al. "Silver Nanoparticles: Synthesis, Characterization, Properties, Applications, and Therapeutic Approaches." International Journal of Molecular Sciences 17.9 (2016): 1534. Web.

Zia-Ul-Haq, Muhammad et al. "Antioxidant Activity of the Extracts of Some Cowpea (Vigna Unguiculata (L) Walp.) Cultivars Commonly Consumed in Pakistan." Molecules 18.2 (2013): 2005-2017. Web. 